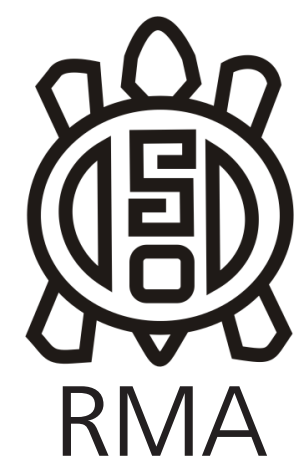

Dossier

\title{
Exploración experimental de los efectos del pisoteo sobre artefactos de obsidiana
}

\author{
Experimental assessment of trampling effects on obsidian artifacts
}

Karen Borrazzo*, Celeste Weitzel** y Antonio Ceraso***

* CONICET, Instituto Multidisciplinario de Historia y Ciencias Humanas (CONICETIMHICIHU). Facultad de Filosofía y Letras, Universidad de Buenos Aires (FFyL - UBA),

Buenos Aires, Argentina. E-mail: kborrazzo@yahoo.com.ar

**CONICET, Área Arqueología y Antropología, Museo de Ciencias Naturales, Necochea, Buenos Aires, Argentina. E-mail: celweitzel@gmail.com

***E-mail: antonioceraso@gmail.com

\begin{abstract}
Resumen
Como continuación de un proyecto destinado a contribuir al conocimiento arqueológico de los efectos de los procesos post-depositacionales sobre los materiales líticos, se realizó una experiencia de pisoteo sobre artefactos de obsidiana. El experimento incluyó el planteo de dos pistas -una sobre sustrato blando y otra sobre sustrato duro- en las que se depositaron artefactos experimentales manufacturados a partir de nódulos de obsidiana procedente de la fuente Huenul (Neuquén, Argentina). Siguiendo la propuesta planteada para evaluar el Potencial para la Fragmentación por Pisoteo (PFP), los datos experimentales son analizados con la técnica de data mining denominada árbol de decisión. Se informan las frecuencias de fractura y las variables métricas artefactuales relevantes en su ocurrencia. Como fuera registrado en experimentaciones previas, se observó que el sustrato duro promueve la ocurrencia de fracturas y contrastamos los espesores máximos de fracturas obtenidos. La incorporación de los datos de obsidiana al árbol de decisión mostró que ciertas materias primas pueden modificar las variables de relevancia y/o su influencia sobre el PFP.
\end{abstract}

Palabras clave: experimentación; obsidiana; potencial para la fragmentación por pisoteo; tafonomía lítica.

\begin{abstract}
This paper presents the results of a trampling experiment on obsidian artifacts conducted as part of a larger archaeological program aimed at assessing the effects of postdepositional processes on lithic artifacts. Flaked artifacts were made from obsidian nodules from Huenul source (Neuquén, Argentina) and were laid on two plots -hard and soft substrate. Here we report the breakage frequency and artifact metric variables relevant for fracture occurrence. Following the methodological proposal to assess the Trampling Fragmentation Potential (TFP), we apply the data mining technique known as decision tree to analyze the experimental obsidian datasets. Results show that fracture ratio is significantly higher on hard substrates, and that the value of maximum fracture thickness shows no variations in obsidian. Finally, including the obsidian dataset to the decision tree showed that certain raw materials may modify the relevant variables and/or their influence on the TFP.
\end{abstract}

Keywords: experimental archaeology; obsidian; trampling fragmentation potential; lithic taphonomy.

\section{Introducción}

La evaluación de los efectos de los procesos postdepositacionales sobre los materiales líticos ha contribuido sustancialmente al estudio y comprensión de los patrones del registro arqueológico (e.g. Dibble, McPherron, Chase, Farrand, y Debénath, 2006; Demeter et al., 2010; Thièbaut, 2010; Bertran, Lenoble, Todisco, Desrosiers, y Sørensen, 2012; Borrazzo, 2016; Carranza Elola y Méndez, 2017). En un trabajo anterior nos hemos abocado específicamente a la evaluación de los efectos del pisoteo, buscando desarrollar elementos para identificar su presencia en los conjuntos líticos (Weitzel,
Borrazzo, Ceraso, y Balirán, 2014). Los resultados allí obtenidos nos llevaron a considerar que las distintas materias primas representadas en el componente lítico, en tanto sus propiedades físico-mecánicas varían, pueden ofrecer respuestas diferentes al ser expuestas a los mismos procesos (Amick y Mauldin, 1997). Con el objetivo de explorar la variación en estas respuestas en los artefactos líticos sujetos a pisoteo, diseñamos un estudio experimental de pisoteo humano sobre artefactos de obsidiana, una materia prima frecuente en el registro arqueológico considerada entre las más frágiles del mundo (Whittaker, 1994). Más específicamente, se buscó evaluar: a) la frecuencia de fracturas por pisoteo 
Tabla 1. Estadística descriptiva de las variables métricas del conjunto experimental incluidas en el árbol de decisión. Todas las medidas en mm. Ref: S/Es: superficie/espesor; L/A/Es: largo/ancho/espesor.

Table 1. Descriptive statistics of the data set metric variables used in the decision tree. All measures in $\mathrm{mm}$. Ref: S/Es: areal thickness; L/A/Es: length/width/thickness.

\begin{tabular}{|l|r|r|r|r|r|}
\hline & \multicolumn{1}{|c|}{ Largo } & \multicolumn{1}{c|}{ Ancho } & \multicolumn{1}{c|}{ Espesor } & \multicolumn{1}{c|}{ S/Es } & \multicolumn{1}{l|}{ LA/Es } \\
\hline N & 129 & 129 & 129 & 129 & 129 \\
\hline Mínima & 16 & 9 & 1 & 41,25 & 0,060 \\
\hline Máxima & 66 & 48 & 21 & 331,5 & 1,222 \\
\hline Media & 31,504 & 22,857 & 6,801 & 114,19 & 0,264 \\
\hline Mediana & 30 & 22 & 6 & 108,65 & 0,211 \\
\hline Varianza & 82,587 & 56,755 & 8,317 & 2539,407 & 0,031 \\
\hline Desvío Stand. & 9,088 & 7,534 & 2,884 & 50,393 & 0,176 \\
\hline
\end{tabular}

en obsidiana, b) la existencia o no de variaciones en los valores del Potencial para la Fragmentación por Pisoteo (PFP) obtenidos en un estudio previo (Weitzel et al., 2014), y c) los espesores máximos registrados en las fracturas por pisoteo de artefactos de obsidiana. Esto permitirá evaluar el PFP de la obsidiana y luego conocer cómo inciden los parámetros de la obsidiana en los valores del PFP general que propusimos a partir de una muestra de artefactos experimentales confeccionados en materias primas más tenaces (Weitzel et al., 2014).

\section{Materiales y métodos}

Los artefactos experimentales fueron confeccionados a partir de la reducción, por percusión directa con percutores líticos, de 9 nódulos de obsidiana procedentes de la fuente Huenul, localizada en la provincia de Neuquén (Barberena et al., 2018). Los nódulos tenían entre 45 y 102 mm de longitud. De los artefactos tallados se seleccionaron 129 lascas buscando la representación de una variedad de tamaños y espesores (Tabla 1). Antes del pisoteo, se fotografió la muestra experimental y se dibujaron los contornos de cada una de las lascas registrando la presencia de fisuras o impurezas, fracturas, retoques, modificaciones asignables a rastros complementarios, etc. Finalmente, se registraron las medidas (largo, ancho y espesor máximo) de cada uno de los artefactos. Con los materiales seleccionados se conformaron dos pistas experimentales, siguiendo los lineamientos de nuestros experimentos previos (Flegenheimer y Weitzel, 2007; Borrazzo, 2011, 2013; Weitzel et al., 2014). La pista sobre sustrato "blando" se estableció sobre sedimento limo arenoso, en el parque Miguel Lillo de Necochea (provincia de Buenos Aires). En ella se sembraron 64 piezas. La pista sobre sustrato duro se instaló en una vereda de baldosas de cemento en un patio particular. En ese caso se sembraron 65 piezas. El pisoteo fue realizado por dos personas (mujeres de $\sim 60 \mathrm{~kg}$ de peso) con calzado de suela blanda (cuero y goma delgada). Las piezas fueron depositadas en cada pista con la cara más convexa hacia abajo (en contacto con sustrato) para maximizar las condiciones de inestabilidad de las piezas. Los dos operadores realizaron 4 series de pasadas, cada una de 10 minutos de duración ( 35 a 50 pasadas dobles por agente cada 10 minutos) en cada pista. Cada 10 minutos se realizó un control de las piezas, se tomaron fotografías y anotaciones.

La muestra de artefactos experimentales utilizada para evaluar el PFP con el árbol de decisión es de 383 piezas, que incluyen los 129 artefactos de obsidiana y las muestras experimentales $(\mathrm{N}=254)$ de los datos analizados en un trabajo previo (Weitzel et al., 2014). Las variables independientes consideradas para el análisis del estado de los artefactos (entero/fragmentado) en este trabajo incluyeron: 1) agente (humano, fauna); 2) dureza del sustrato (duro, blando); 3) materia prima del artefacto (obsidiana, ortocuarcita, lutita, dacita y roca silicificada); 4) largo máximo del artefacto (L); 5) ancho máximo del artefacto (A); 6) espesor máximo del artefacto (Es); y dos índices: 7) largo máximo/ancho máximo/espesor máximo (L/A/Es) y 8) la superficie del artefacto (el producto de L por A) dividido por el espesor máximo (S/Es). La estadística descriptiva (Tabla 1) y los test fueron realizados con el programa past 3.22. El árbol de decisión se realizó con el programa R 2.11.0 (R Developement Core Team, 2011).

\section{Resultados}

En primer lugar, se observó que la frecuencia de fragmentación de los artefactos de obsidiana fue mayor en la pista sobre sustrato duro (43 de 65 fracturadas, 66, 15\%) que sobre sustrato blando (24 de 64 fracturadas, 37,5\%). Este resultado es similar a los obtenidos en experimentos con diferencias en la dureza de los sustratos, realizados tanto con obsidiana (GiffordGonzález, Damrosch, Damrosch, Prior y Thunen, 1985; Nielsen, 1991), como con otras materias primas (e.g McBrearty, Bishop, Plummer, Dewar y Conard, 1998; Weitzel, 2010). Cabe destacar que la diferencia en este caso es significativa ( $p=0,0015$, test exacto de Fisher). 
En cuanto al espesor de la sección de las fracturas, los resultados muestran que no hay diferencias significativas entre los espesores de las piezas fracturadas en la pista blanda y la pista dura (Mann-Whitney $U=432, p=0,2702$ ). Las medias de los espesores de las fracturas rondan en ambos casos los $3 \mathrm{~mm}$ y el espesor máximo observado en las fracturas de ambas pistas es virtualmente idéntico (7 vs 7,1 mm) (Tabla 2). Cabe destacar que este es el mismo valor máximo que hemos obtenido en los otros 12 experimentos con materias primas más tenaces que la obsidiana (Weitzel et al., 2014, Borrazzo, 2017, 2019).

Las fracturas múltiples son las más frecuentes $(35,82 \%)$ y se destacan las fracturas cortas y continuas sobre los sectores muy delgados de las lascas, cerca del borde, que presentan formas rectas y cóncavas o forma de "media luna", que son fracturas típicas de pisoteo (Clemente Conte, 1997; Weitzel, 2010). Entre las fracturas múltiples se registra la presencia de fracturas con patrón radial (6\%); una de ellas es resultado de una fractura compresiva, con rasgos que podrían confundirse con los de una fractura intencional. Otras roturas frecuentes fueron fracturas simples marginales, que también se dan en mayor número sobre los sectores más delgados de las lascas (aunque escasas, las hay también más espesas). Éstas se presentan en posición oblicua, transversal, distal, proximal y lateral. Las diferenciamos de las múltiples con estas características porque se trata de una sola fractura sobre la pieza. Les siguen fracturas de posición transversal, tanto en sectores delgados como en sectores más espesos, cerca del centro de las piezas. En menor medida registramos fracturas del talón, fracturas longitudinales (una de ellas tiene la ubicación de una fractura de tipo split) y fracturas de posición oblicua. Los tipos registrados incluyen fracturas de flexión y, en menor medida, compresivas (que son fracturas que se inician en el lugar en el que se ejerció la presión y que presentan evidencias del punto de inicio de la fractura sobre una de las caras). Los tipos de fractura y su localización en la pieza son similares a los registrados en experiencias previas (Weitzel, 2010); las diferencias más destacadas recaen en la presencia de fracturas compresivas en la obsidiana y en la distribución similar de fracturas de posición múltiple en ambas pistas de obsidiana, dado que en el experimento con ortocuarcitas (Weitzel, 2010) las fracturas múltiples se asocian a un sustrato duro.

Con respecto al potencial de fragmentación, en un trabajo anterior (Weitzel et al., 2014) aplicamos el método de data mining denominado árbol de decisión para evaluar el PFP de un artefacto lítico. En aquella oportunidad usamos los datos de cinco experimentos con ocho pistas experimentales diferentes con sustratos de diversas características y durezas, realizados con materias primas mayormente tenaces (ortocuarcita, riolita, lutita, rocas silicificadas), y distintos agentes de
Tabla 2. Estadística descriptiva de los espesores de las fracturas producidas por pisoteo registradas en los artefactos experimentales de obsidiana.

Table 2. Descriptive statistic of fracture thickness recorded on obsidian artifacts after trampling experiments.

\begin{tabular}{|l|r|r|}
\hline & $\begin{array}{c}\text { Espesor de fractura } \\
(\mathrm{mm})\end{array}$ & $\begin{array}{c}\text { Espesor de fractura } \\
(\mathrm{mm})\end{array}$ \\
\hline $\mathbf{N}$ & \multicolumn{1}{|c|}{ Sustrato Duro } & Sustrato Blando \\
\hline Mínima & 1 & 24 \\
\hline Máxima & 7,1 & 7 \\
\hline Media & 3,184 & 2,771 \\
\hline Varianza & 2,641 & 2,283 \\
\hline Desvío Estándar & 1,625 & 1,511 \\
\hline Mediana & 3,00 & 2,50 \\
\hline
\end{tabular}

pisoteo (humanos, animales de pequeño y gran porte). En el árbol de decisión generado con esos datos, el modelo seleccionó como la variable más importante para explicar el estado (entero o fragmentado) de los artefactos a la relación entre la superficie y el espesor de las piezas (S/Es). Luego de seleccionar el índice S/Es, el árbol muestra otras variables métricas que inciden en el potencial de fragmentación de los artefactos, pero en ningún caso se seleccionaran otros atributos de las piezas (e.g. materia prima) o variables contextuales (e.g. dureza del sustrato). Además, la variable S/Es nos mostraba un valor $(172,28)$ que separaba la muestra experimental de acuerdo al potencial para la fragmentación por pisoteo de cada artefacto (i.e. si era pasible o no de ser fracturado por pisoteo). De acuerdo con ese resultado, aquellos artefactos cuyo índice S/Es fuera mayor o igual a 172,28 tendrían un alto PFP y serían más propensos a fracturarse al estar expuestos a situaciones de pisoteo. Ya en esa oportunidad pensamos que teníamos que evaluar el caso de la obsidiana, que ofrece propiedades físicas diferentes, lo que podía modificar el valor numérico del índice o que podía transformar a la materia prima en una variable de mayor importancia para el árbol de decisión.

Al incorporar a la base de datos preexistente los nuevos datos obtenidos en la experiencia de pisoteo de los artefactos de obsidiana y correr nuevamente los análisis del árbol de decisión, la variable "materia prima" fue seleccionada por el árbol como la más importante para explicar el estado (entero o fragmentado) de las piezas. La Figura 1 es una representación gráfica del nuevo árbol obtenido. Como puede observarse en ella, el atributo materia prima genera una primera divergencia en dos ramas independientes: hacia la derecha se seleccionaron los artefactos de obsidiana mientras que a la izquierda 
quedaron agrupadas las demás materias primas que usamos en otros experimentos. Esta segunda rama repite la estructura del árbol de decisión que obtuvimos y publicamos previamente (Weitzel et al., 2014). En la rama de la derecha en la Figura 1, que representa exclusivamente a los artefactos experimentales de obsidiana, vemos que la segunda variable de importancia para explicar el estado de las piezas es la "dureza del sustrato". Este elemento contrasta con lo registrado en los artefactos experimentales de las demás materias primas, entre las que la dureza del sustrato no ha sido una variable seleccionada por el árbol como influyente para explicar el estado de las piezas (Weitzel et al., 2014). A partir del nodo "dureza del sustrato" ("sustrato" en Figura 1) comienzan a seleccionarse los atributos métricos y sus índices, siendo S/Es el primero elegido por el árbol. Por último, la superficie artefactual ( $\mathrm{S}=$ largo máximo por ancho máximo) y el largo máximo de las piezas (L) son dos atributos señalados como relevantes por el árbol de decisión para definir el estado de los artefactos de obsidiana sujetos a pisoteo sobre sustratos blandos (Figura 1).

\section{Discusión y conclusiones}

Sintetizamos aquí algunas ideas, hipótesis y reflexiones para seguir trabajando.

El experimento de pisoteo sobre artefactos de obsidiana mostró que los patrones de fractura son similares a los observados en otras materias primas, pero ocurren con más frecuencia (aunque baja) las fracturas compresivas que dejan sobre la superficie de fractura rasgos que indican su origen sobre alguna de las caras de la pieza y que podrían eventualmente confundirse con fracturas intencionales. Por otra parte, al igual que lo registrado en otros experimentos, el índice de fragmentación aumenta con la dureza del sustrato (e.g. Gifford-González et al, 1985; Nielsen, 1991; McBrearty et al., 1998; Weitzel, 2010).

Con respecto al estudio del PFP, dos observaciones resultan relevantes. Por un lado, a pesar de su mayor fragilidad, el espesor máximo de la fractura obtenida por pisoteo con agentes de $\sim 60 \mathrm{~kg}$ en artefactos de obsidiana es igual a

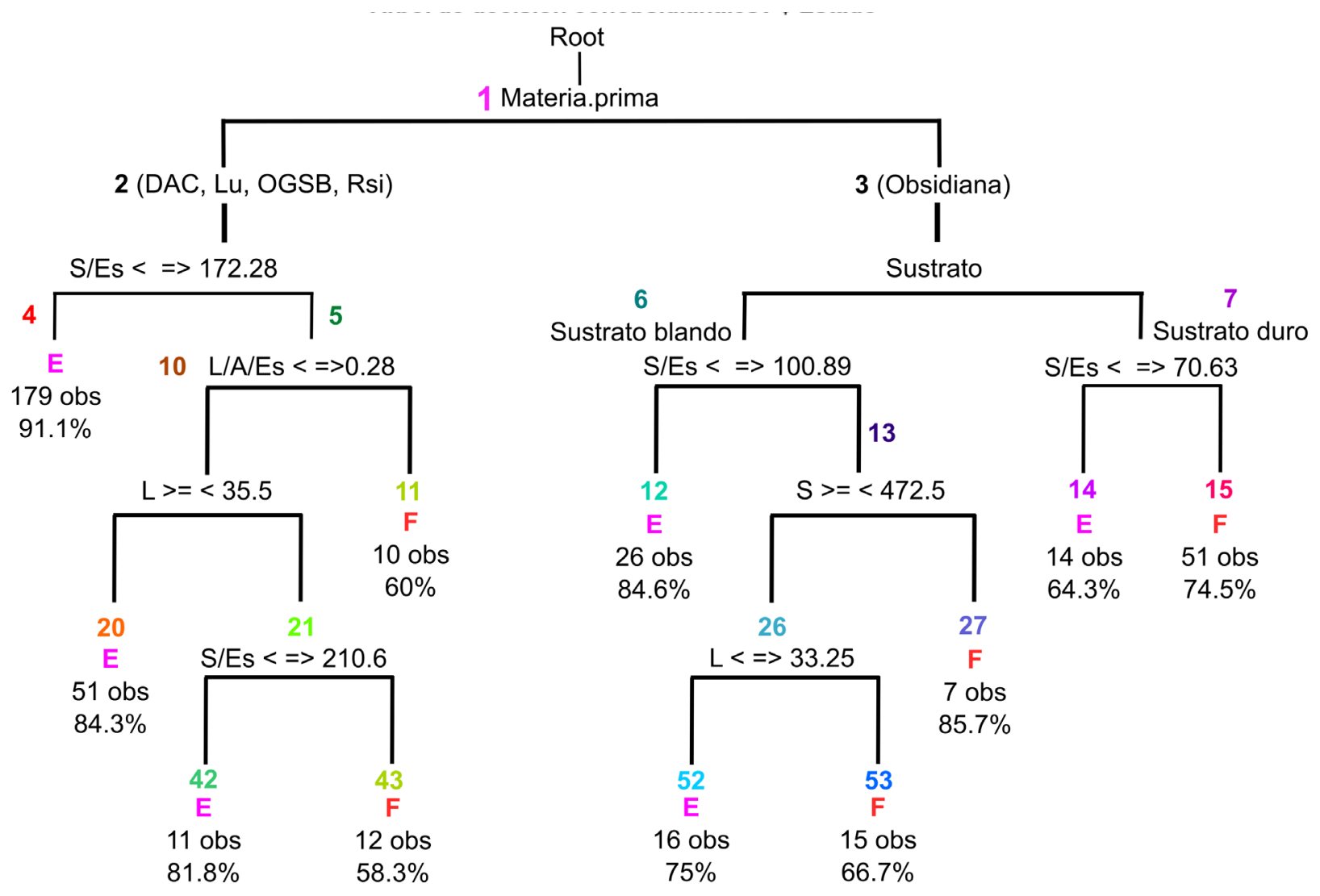

Figura 1. Árbol de decisión para el estado (entero/fragmentado) de los artefactos experimentales sujetos a pisoteo incluyendo los artefactos de obsidiana y los de otras materias primas de experimentos previos. Ref.: DAC: Dacita; Lu: Lutita; OGSB: Ortocuarcitas Grupo Sierras Bayas; Rsi: Roca silicificada; S: Superficie; Es: Espesor; L: Largo; A: Ancho; E: Entero; F: Fragmentado; obs: observaciones.

Figure 1. Decision tree for the condition (whole/broken) of trampled experimental artifacts, including obsidian artifacts and artifacts of different raw material from previous experiments. Ref.: DAC: Dacite; Lu: Shale; OGSB: Sierras Bayas Group orthoquartzites; Rsi: Silicified rocks; S: Area; Es: Thickness; L: Length; A: Width; E: Whole; F: Broken; obs: observations. 
aquel que hemos registrado hasta el momento en otras experiencias de pisoteo con materias primas más tenaces ( $\sim 7 \mathrm{~mm})$. Por otro lado, el análisis con el árbol de decisión mostró que la obsidiana exhibe un comportamiento que la separa completamente de las demás materias primas examinadas hasta el momento. Como anticipamos, su inclusión en la muestra analizada con el árbol de decisión produjo la selección de una nueva variable (la materia prima) como la más relevante para evaluar el PFP con nuevos datos. En esa submuestra de obsidiana, primero el sustrato y luego los atributos métricos de las piezas tienen incidencia sobre su probabilidad de fractura. Por otra parte, cabe destacar que el modelo sigue seleccionando sobre otras al índice S/E como variable influyente en la ocurrencia de fracturas. El árbol de decisión brinda un modelo para explicar el PFP, sujeto a cambios en función de la incorporación de nuevos datos. En este caso, la incorporación de la obsidiana generó una rama única para esta materia prima, construida en función de las variables sometidas a análisis. Nuevas variables definidas para atributos específicos de esta materia prima o la incorporación de variables contextuales adicionales podrán complejizar y enriquecer el modelo.

Buenos Aires, 26 de agosto de 2019.

\section{Agradecimientos}

A Ramiro Barberena, quien proveyó la materia prima para el estudio experimental. A los editores del volumen. Esta investigación ha sido financiada por CONICET y ANPCYTFONCyT.

\section{Referencias citadas}

Amick, D. S., \& Mauldin, R. P. (1997). Effects of Raw Material on Flake Breakage Patterms. Lithic Technology, 22(1), 18-32.

Barberena, R., Fernández, M.V., Rughini, A.A., Borrazzo, K., Garvey, R., Lucero, G., Della Negra, C., Romero Villanueva, G., Durán, V., Cortegoso, V., Giesso, M., Klesner, C., MacDonald, B.L., \& Glascock, M.D. (2018). Deconstructing a Complex Obsidian 'Source-scape': A Geoarchaeological and Geochemical Approach in Northwestern Patagonia. Geoarchaeology: an International Journal, 1-12. https://doi.org/10.1002/ gea.21701.

Bertran, P., Lenoble, A., Todisco, D., Desrosiers, P., \& Sørensen, M. (2012). Particle size distribution of lithic assemblages and taphonomy of Palaeolithic sites. Journal of Archeological Science, 39, 3148-3166.

Borrazzo, K. (2011). Tafonomía lítica en la estepa patagónica: experimentación y registro arqueológico de superficie. En L.A. Borrero \& K. Borrazzo (Comps.), Bosques, Montañas y cazadores: investigaciones arqueológicas en Patagonia Meridional (p.127-153). Buenos Aires, CONICET-IMHICIHU.

Borrazzo, K. (2013). Tafonomía lítica y modelo de la dinámica eololacustre del norte de la bahía San Sebastián (Tierra del Fuego, Argentina). Revista Comechingonia, 17(1), 149-169.

Borrazzo, K. (2016). Lithic Taphonomy in desert environments: contributions from Fuego-Patagonia (Argentina). Quaternary International, 422, 18-29. https://doi.org/10.1016/j.quaint.2015.12.012.

Borrazzo, K. (2017). El rol de los criterios tecnomorfológicos en el reconocimiento de pseudoartefactos. Libro de resúmenes de las $X$ Jornadas de Arqueología de la Patagonia, (p. 21). Puerto Madryn, CENPAT-CONICET.

Borrazzo, K.(2019). Expanding the scope of Actualistic Taphonomy in Archaeological Research. En S. Martínez, A. Rojas, y F. Cabrera (Eds.) Actualistic Taphonomy in South America, (pp 221-242). Nueva York, Topics in Geobiology 48, Springer, Cham.

Carranza Elola, J. J., \& Méndez, C. (2017). Lithic Taphonomy at the Quebrada de Quereo Site: addressing the problem of anthropogenic ambiguity in Late Pleistocene Assemblages. En J. Alberti, K. Borrazzo, S. Buscaglia, A. Castro Esnal, A. Elías, \& N. Franco (Eds.), 11th International Symposium on Knappable Materials "From toolstone to stone tools" (p. 199). Buenos Aires: IMHICIHU-CONICET.

Clemente Conte, I. (1997). Los instrumentos líticos de Túnel VII: una aproximación etnoarqueológica. Madrid: CSIC y UAB.

Demeter, F., Edoumba, E., Duringer, P., Bacon, A-M., Sytha, P., Bano, M., Laychour, V., Cheangleng, M., \& Sari, V. (2010). Reinterpretation of an archaeological pebble culture from the Middle Mekong River valley, Cambodia. Geoarchaeology, 25(1), 75-95

Dibble, H. L., McPherron, S. J. P., Chase, P.G., Farrand, W.R., \& Debénath, A. (2006). Taphonomy and the concept of Paleolithic cultures: the case of the Tayacian from Fontéchevade. PaleoAnthropology, 1-21.

Flegenheimer, N., \& Weitzel, C. (2007). Caminar sobre piedras: los artefactos fracturados de Cerro El Sombrero. En XVI Congreso Nacional de Arqueología Argentina (Tomo III, pp. 263-267). Jujuy: Universidad Nacional de Jujuy.

Gifford-Gonzalez, D. P., Damrosch, D. B., Damrosch, D. R. Prior, J., \& Thunen, R. (1985). The Third Dimension in Site Structure: an Experiment in Trampling and Vertical Dispersal. American Antiquity, 50(4), 803-818. 
McBrearty, S., Bishop, L., Plummer, T., Dewar, R., \& Conard, N. (1998). Tools underfoot: human trampling as an agent of lithic artifact edge modification. American Antiquity, 63(1), 108-122.

Nielsen, A. E. (1991). Trampling the Archaeological Record: An Experimental Study. American Antiquity, 56(3), 483-503.

R Development Core Team (2011). R: A language and environment for statistical computing, reference index version 2.2.1. URL. R Foundation for Statistical Computing, Vienna, Austria. http://www.R-project.org

Thiébaut, C. (2010). Denticulate Mousterian: myth or reality? En J. M. Burdukiewicz, \& A. Wisniewski (Eds.), Middle Palaeolithic Human Activity and Palaeoecology:
New Discoveries and Ideas (pp. 345-386). Wroclaw: Wydawnictwo Uniwersytetu Wrocławskiego.

Weitzel, C. (2010). El estudio de los artefactos formatizados fracturados. Contribución a la comprensión del registro arqueológico y las actividades humanas (Tesis doctoral no publicada). Facultad de Filosofía y Letras, Universidad de Buenos Aires, Argentina.

Weitzel, C., Borrazzo, K., Ceraso, A., \& Balirán, C. (2014). Trampling fragmentation potential of lithic artifacts: an experimental approach. Intersecciones en Antropología, 15(Suplemento 1), 97-110.

Whittaker, J.C., 1994. Flintknapping: Making and Understanding Stone Tools. Austin: University of Texas Press. 